Research Article

\title{
A Novel Shock Absorber with the Preload and Global Negative Stiffness for Effective Shock Isolation
}

\author{
Zecui Zeng $\mathbb{D}^{1},{ }^{1}$ Lei Zhang $\left(\mathbb{D},{ }^{1,2}\right.$ and Ming Yan $\mathbb{D}^{1}$ \\ ${ }^{1}$ School of Mechanical Engineering, Shenyang University of Technology, Shenyang 110870, China \\ ${ }^{2}$ Naval Research Academy, Beijing 100161, China \\ Correspondence should be addressed to Lei Zhang; freda_zl@126.com
}

Received 10 August 2020; Revised 5 February 2021; Accepted 15 February 2021; Published 25 February 2021

Academic Editor: Jean-Jacques Sinou

Copyright (c) 2021 Zecui Zeng et al. This is an open access article distributed under the Creative Commons Attribution License, which permits unrestricted use, distribution, and reproduction in any medium, provided the original work is properly cited.

A novel shock absorber with the preload structure and global negative stiffness is proposed for the shock isolation of sensitive systems. The novel shock absorber is composed of a linear spring and permanent magnet sets. The preload force and negative stiffness region are related to the attractive force between permanent magnet sets. The aim of this paper is to investigate the shock isolation performance of the novel shock absorber. Firstly, a static analysis of the novel shock absorber is carried out. Secondly, the motion stability of the NSA is analyzed by the Jacobian Matrix and the shock response is calculated numerically compared with the conventional preload structures. Finally, the shock test of the novel shock absorber is completed to verify the above results. It is found that the novel shock absorber could be advantageous in improving shock isolation in terms of relative displacement and absolute acceleration compared with conventional preload structures.

\section{Introduction}

The sensitive systems, such as the inertial navigation system, are particularly sensitive to the vibration and shock inputs from the foundation [1]. These inputs often lead to errors in the inertial sensors of the navigation system, which could result in velocity and heading errors. Moreover, the sensitive systems can lose function under severe shocks caused by various intensive impulsive loads [2]. To protect sensitive systems, a shock absorber should be mounted between the host systems and the foundation. However, a conventional shock absorber is not enough to ensure the sensitive systems work well in a complex environment. For example, during spacecraft launch, a preload structure should be used to maintain the sensitive systems support structure in a fixed relationship with the spacecraft, and it can be released to allow relative movement after launch [3]. Thus, a shock absorber with preload structure is effective in protecting the sensitive systems in such a complex environment.

The nonlinearity of the preload structure has been investigated and the vibration characteristics can be analyzed by the harmonic balance method [4]. Heimbs studied the shock test and modeling of carbon fibre-reinforced plastic plates with compression preloading at a low-speed shock and found that the preloaded carbon fibre-reinforced plates have lower energy absorption effects than the free carbon fibre-reinforced plates [5]. Garcia studied the shock attenuation characteristics of a gasket with an initial compressive load under repeated shock [6]. However, the elastic elements of the preload structures mentioned above usually have positive stiffness. The negative stiffness elements were often used to obtain low-frequency or quasi-zero stiffness structures [7]. A cam structure, a precompression spring structure, and a magnet were used to generate negative stiffness for obtaining a quasi-zero stiffness structure [8]. A shock absorber with a negative stiffness element has advantages compared with linear stiffness [9]. However, the vibration absorber with a negative stiffness element mentioned above generally is global positive stiffness or quasi-zero stiffness, so that the force response of the sensitive systems increases as the relative displacement response increasing.

This paper proposed a novel shock absorber (NSA) with the preload structure and global negative stiffness. The NSA not only has the preload function but also can ensure the 
force response would not exceed the preload force with the deformation increasing due to global negative stiffness. The aim of this paper is to study the shock isolation performance of the NSA. In Section 2, the prototype of the NSA is proposed and the static analysis of the NSA is studied. In Section 3, the dynamic stability analysis of the NSA is investigated by solving the eigenvalue equation of the system. The shock response of the NSA is calculated numerically compared with a preload structure with positive stiffness (PSPS) and a linear stiffness system (LSS). In Section 4, a prototype of the NSA is fabricated and the shock test is completed to test the shock isolation performance of the NSA.

\section{Static Analysis}

A preload structure could improve the robustness of the initial equilibrium position and protect equipment from severe shock excitations. Generally, a preload structure was composed of a precompressed or pretensioned elastic element. In this study, a pair of permanent magnets are used to generate the preload force.

The schematic diagram of the NSA considered in this paper is shown in Figure 1(a). It comprises a mass (5), supported by a linear spring (6) $k$, guiding rod (8), and four permanent magnet sets (1-1), (1-2), (2-1), and (3-1). The magnet sets (1-1) and (1-2) are grouped into arrays on the suspended plane (1), magnet set $(2-1)$ is on the fixed plane (2), and magnet set (3-1) is on the moved plane (3). The mass (5) is the sensitive system to be isolated from the shock excitation. Four permanent magnet sets were used to generate the negative stiffness.

The permanent magnet sets (1-1) and (3-1) are separated when mass moves down as shown in Figure 1(b). Permanent magnet sets (1-2) and (2-1) are separated when mass moves up as shown in Figure 1(c). The relative displacement $x$ is equal to $y$ subtract $z$. The gap $h$ between the separated permanent magnets is equal to $|x|$. The Vakoun studied the force between the cylindrical permanent magnets and derived the relevant calculation formula and experimental verification [10]. The attractive force between two magnet sets is the red dash-dot line shown in Figure 2, and it can be written as [10]

$$
F_{m}=-8 \pi K_{d} R^{2} \int_{0}^{+\infty} \frac{J_{1}^{2}(q)}{q^{2}} \sin h(q \tau)^{2} e^{-q \zeta} \mathrm{d} q .
$$

Due to the fact that the magnet pairs analyzed in this paper were separated with a certain distance as shown in Figure 1(a), the interference between magnet pairs could be ignored. Thus, it is assumed that the attractive force of $n$ pairs of magnets is $n$ times larger than a single pair of magnets. And this assumption was verified by static testing in Section 4 as shown in Figure 3(d). Here, to simplify the following calculation process, an exponential function is used to fitting equation (1):

$$
F_{m} \approx n A e^{(-x / \mu)}
$$

where $n$ represents the number of permanent magnets in arrays and $A$ and $\mu$ are the fitting parameters. It is found that the attraction force $F_{\mathrm{m}}$ is closed to zero when the gap increases to a certain value and the attraction force is maximum when the gap $h$ is zero. The resilient force $F_{\mathrm{L}}$ of the linear spring is the blue dot line shown in Figure 2. The linear spring is used to make the mass back to the initial equilibrium position because the attractive force $F_{\mathrm{m}}$ of the magnet sets is unable to support the mass when the gap $h$ is large enough. The equivalent force $F_{\mathrm{k}}$ of the NSA is the product of $F_{\mathrm{m}}$ and $F_{\mathrm{L}}$ as the green solid line shown in Figure 2. The equivalent resilient force is expressed as

$$
F_{k}=F_{L}+F_{m}=k x+n A e^{(-x / \mu)}, \quad x>0
$$

In Figure 2, it is shown that the NSA has a preload force $F_{\max }$ at the equilibrium position, which is generated by permanent magnet sets. Besides, it reveals that there are a negative stiffness region and a positive stiffness region in the force-displacement curve of the NSA. Equation (3) could transform into a dimensionless form:

$$
\widetilde{F}_{k}=\widetilde{F}_{L}+\widetilde{F}_{m}=u+\widetilde{A} e^{(-u / \mu)},
$$

where

$$
\begin{aligned}
\widetilde{F}_{L} & =\frac{F_{L}}{k x_{\max }}, \\
u & =\frac{x}{x_{\max }} \\
\widetilde{F}_{m} & =\frac{n A e^{(-u / \mu)}}{k x_{\max }}, \\
\widetilde{A} & =\frac{n A}{k x_{\max }}, \\
e^{(-u / \mu)} & =\left(e^{(-x / \mu)}\right)\left(1 / x_{\max }\right) .
\end{aligned}
$$

The dimensionless stiffness of the NSA can be obtained by differentiating equation (4) with respect to $\tilde{x}$ :

$$
\widetilde{K}=1-\frac{\widetilde{A}}{\mu} e^{(-u / \mu)} .
$$

The above relationship gives the value of the dimensionless equivalent force or stiffness of the NSA as a function of the preload amplitude $\widetilde{A}$, the relative displacement $\mu$, and the fitting parameter $\mu$. The influence of these parameters on the values of the $\widetilde{F}_{k}$ and $\widetilde{K}$ is shown in Figures 4 and 5 , respectively. In Figure 4(a), it is revealed that the values of $\widetilde{F}_{k}$ change profoundly depending upon the value of $\widetilde{A}$ at the equilibrium position. It shows that the shape of the dimensionless resilient force $\widetilde{F}_{k}$ changes with different values of $\mu$ shown in Figure 4(b). The influence of the preload amplitude $\widetilde{A}$ and the fitting parameter $\mu$ on the $\widetilde{K}$ are shown in Figures 5(a) and 5(b). It can be found that the stiffness of the NSA is negative around the equilibrium position and it decreases with the $\widetilde{A}$ or $\mu$ increasing. 

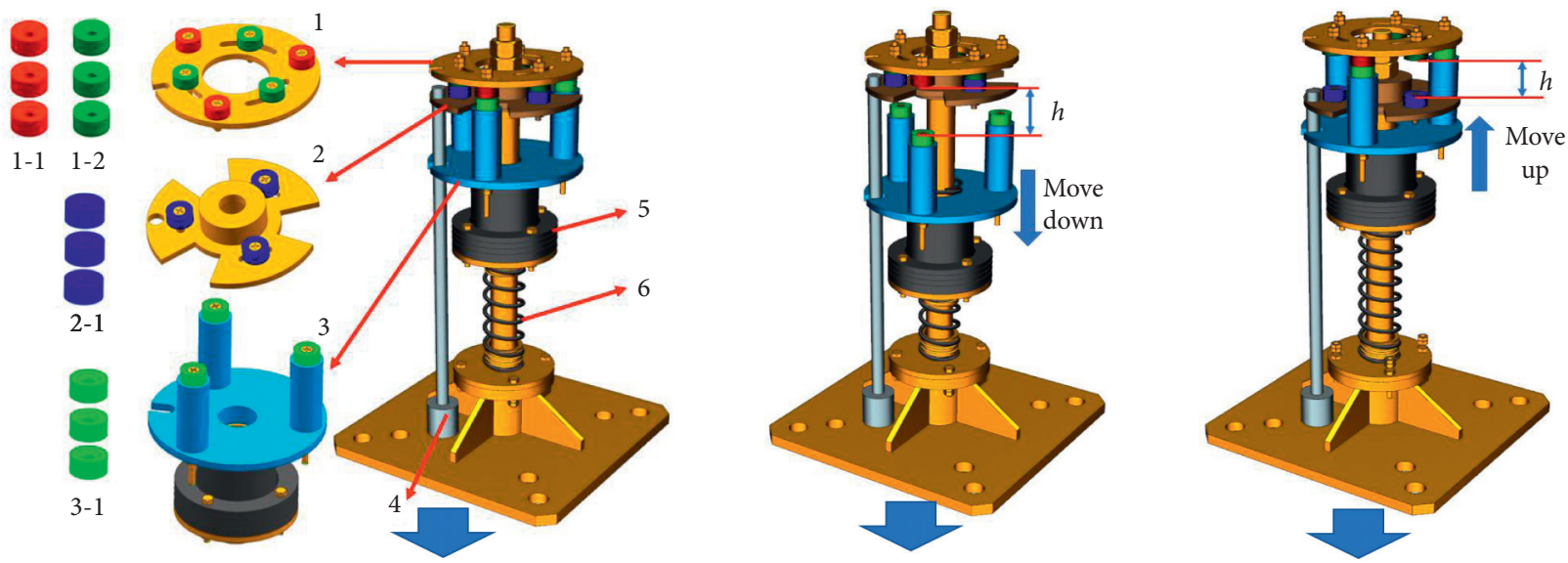

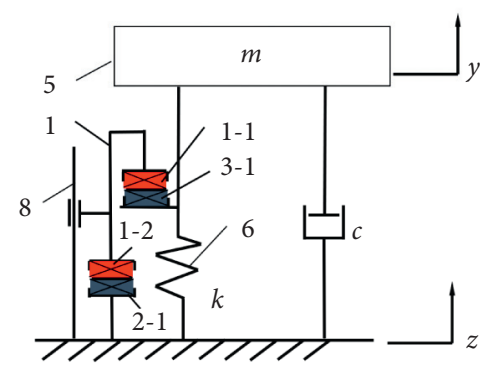

(a)

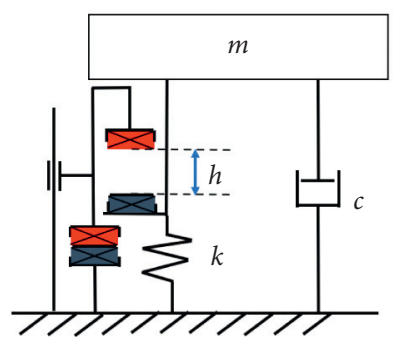

(b)

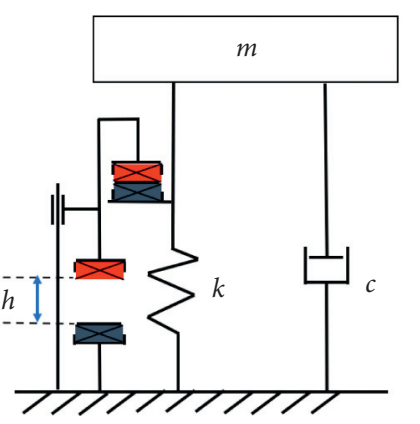

(c)

FIgURE 1: Modeling of the NSA prototype as a spring-mass system.

\section{Dynamic Analysis}

In this section, the dynamic behavior of the NSA is analyzed, including the motion stability, shock response, and the advantages of the NSA compared to other preload structures.

3.1. Problem Formulation. In Figure 1, the equation of motion of the NSA can be given by

$$
m \ddot{x}+c \dot{x}+k x+n A e^{(-x / \mu)}=-m \ddot{z},
$$

where $x=y-z$.

To investigate the shock isolation performance of the NSA, several types of shock excitation types have been considered as the base excitation [11]. Here, the time-domain shock excitation wave presented in BV043/85 standard is adopted as shown in Figure 6 [12].

The shock excitation can be expressed as

$$
m \ddot{z}= \begin{cases}F \sin w t & t<t_{1}, \\ -\frac{F}{n_{1}} \sin n_{1} w\left(t-t_{1}\right), & t_{1}<t<t_{2}, \\ 0, & t_{2}<t .\end{cases}
$$

Equation (7) can transform into a dimensionless form:

$$
u^{\prime \prime}+2 \zeta u^{\prime}+u+\widetilde{A} e^{-(u / \mu)}=-\widetilde{F}(\Omega \tau)
$$

where

$$
\begin{aligned}
w_{n} & =\sqrt{\frac{k}{m},} \\
\zeta & =\frac{c}{2 \sqrt{k m}}, \\
\tau & =w_{n} t, \\
u & =\frac{x}{x_{\max }}, \\
\widetilde{A} & =\frac{n A}{k x_{\max }}, \\
\widetilde{F} & =\frac{m \ddot{z}}{k x_{\max }} \\
\Omega & =\frac{w}{w_{n}}, \\
e^{(-u / \mu)} & =\left(e^{(-u / \mu)}\right)^{\left(1 / x_{\max }\right)} .
\end{aligned}
$$

The motion stability of the system can be assessed by means of a "stability criterion" [13]. By replacing external load with the initial condition, the free vibration of the preload structure is expressed as 


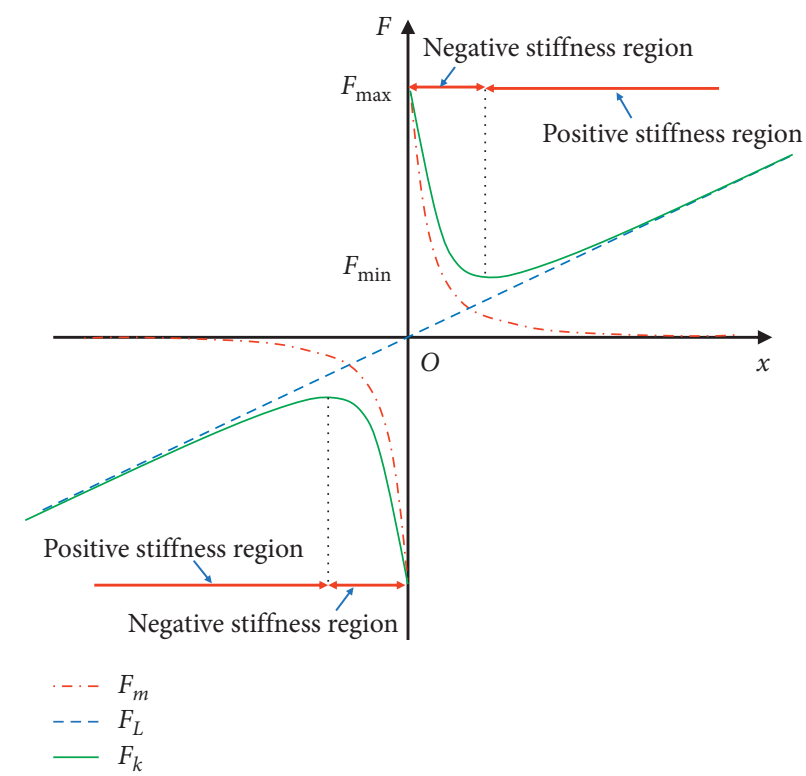

FIGURE 2: The equivalent force-displacement curve of $(\mathrm{F})_{\mathrm{k}}$.

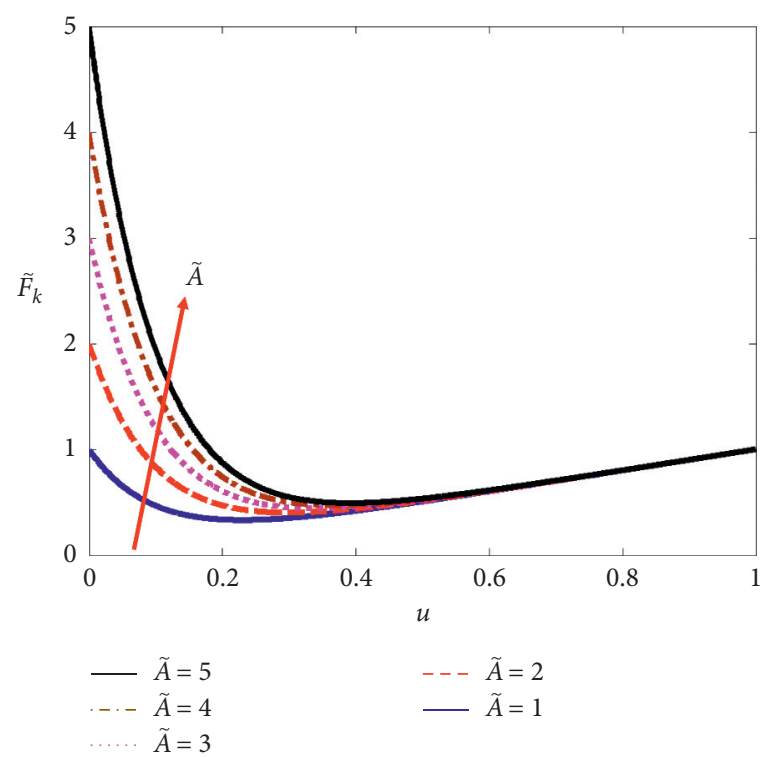

(a)

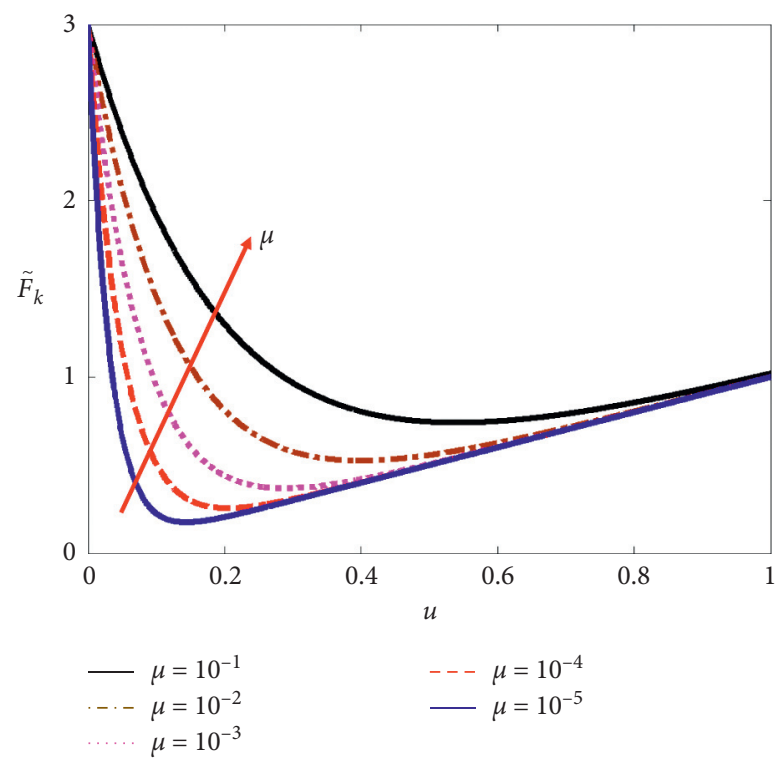

(b)

FIgure 3: Schematic diagram of the static test. (a) The static test of three magnet pairs. (b) The static test of a single magnet pair. (c) The static test of linear springs. (d) The static test curve of magnet sets. (e) The static test curve of linear springs. (f) The static test curve of the NSA. (g) The static test of the NSA.

$$
u^{\prime \prime}+2 \zeta u^{\prime}+u+\widetilde{A} e^{(-u / \mu)}=0
$$

And the initial condition is $u(\tau=0)=u_{0}$, $u^{\prime}(\tau=0)=u_{0}^{\prime}$. The dynamic analysis equation of the system is expressed as

$$
\begin{aligned}
& u^{\prime}=f_{1}(u, q)=q, \\
& \dot{q}=f_{2}(u, q)=-2 \zeta q-u-\widetilde{A} e^{(-u / \mu)},
\end{aligned}
$$

where $x$ and $y$ are the status terms of the system. Based on equations (3) and (4), the Jacobian matrix of the system is expressed as

$$
J=\left|\begin{array}{ll}
\frac{\partial f_{1}}{\partial u} & \frac{\partial f_{1}}{\partial q} \\
\frac{\partial f_{2}}{\partial u} & \frac{\partial f_{2}}{\partial q}
\end{array}\right|
$$




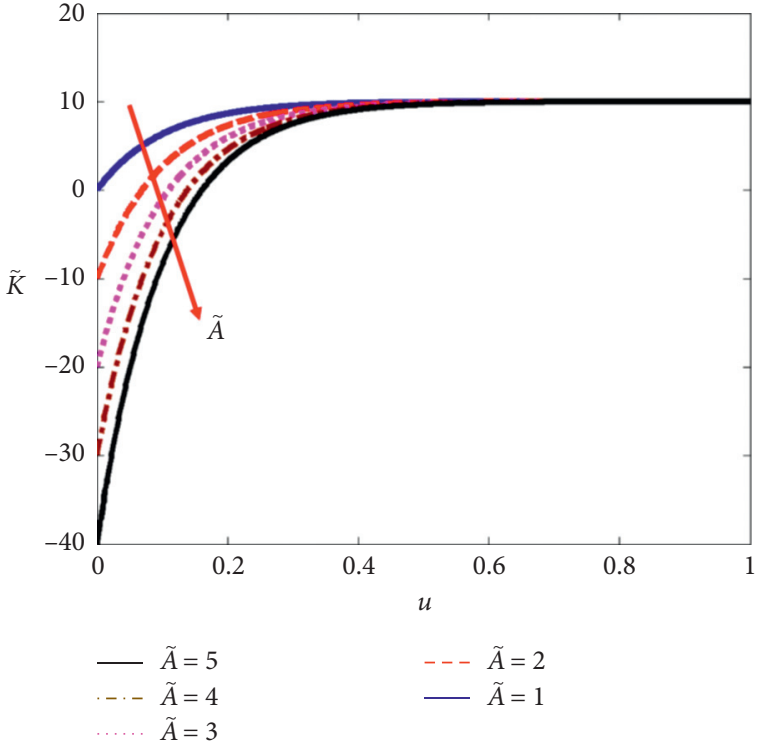

(a)

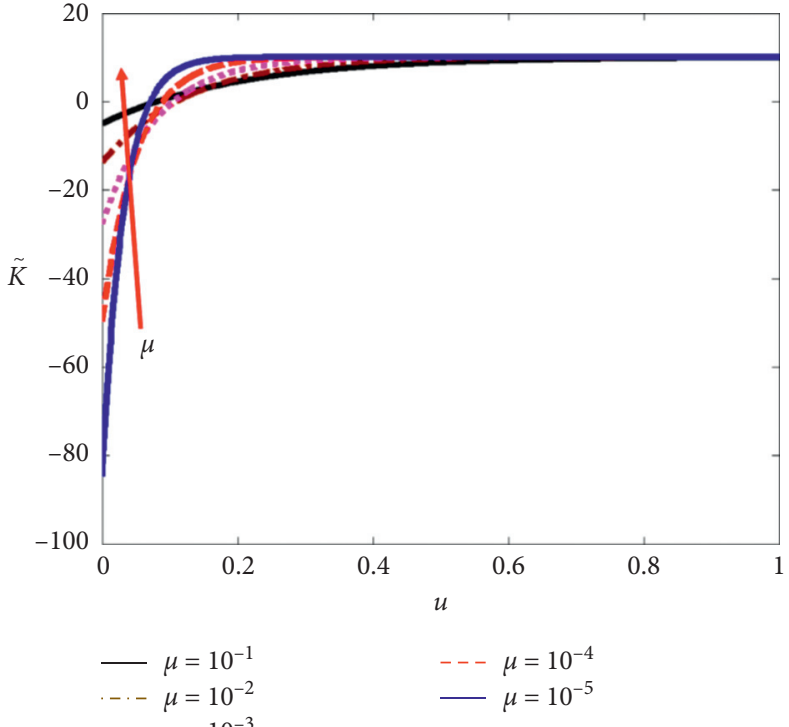

(b)

FIgURE 4: The influence of the preload amplitude $\widetilde{A}$ and the fitting parameter $\mu$ on the $\widetilde{F}_{k}$. (a) Different values of the parameter $\widetilde{A}$. (b) Different values of the parameter $\mu$.

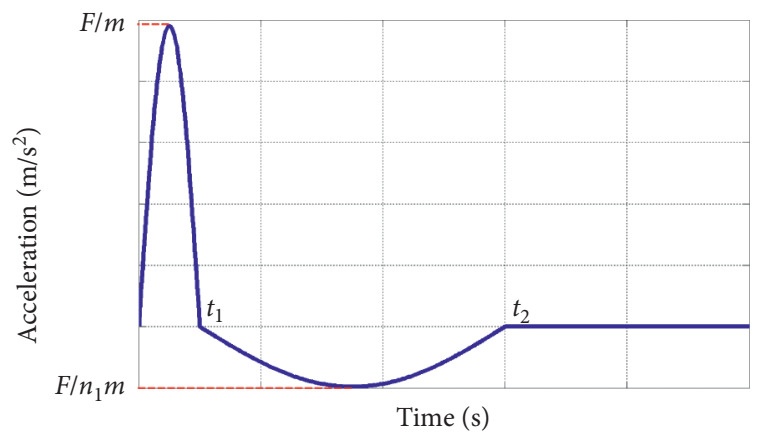

Figure 5: The influence of the preload amplitude $\widetilde{A}$ and the fitting parameter $\mu$, on the $\widetilde{K}$. (a) Different values of the parameter $\widetilde{A}$. (b) Different values of the parameter $\mu$.

And the eigenvalue equation of the system is

$$
|A-\lambda I|=0
$$

According to equations (6) and (7), the eigenvalue equation of the system is expressed as

$$
\lambda^{2}+2 \zeta \lambda+1-\frac{1}{\mu} \tilde{A} e^{(-u / \mu)}=0
$$

By solving equation (14), the eigenvalue expression is obtained:

$$
\lambda_{1,2}=\frac{-2 \zeta \pm \sqrt{(2 \zeta)^{2}-4\left(1-(1 / \mu) \widetilde{A} e^{(-u / \mu)}\right)}}{2}
$$

where $\zeta, \widetilde{A}, \mu$, and $u$ are positive values here. According to the stability criterion, the real parts of $\lambda$ must be negative for stability [13]. Hence, the following criteria can be established to ensure stability:

$$
1-\frac{1}{\mu} \tilde{A} e^{(-u / \mu)}>0
$$

From equation (18), it can be seen that the NSA has a microunstable range around the equilibrium position because of the negative stiffness region. According to equations (12) and (13), a phase diagram of the NSA with different $\% A$ and $\mu$ could be obtained as shown in Figure 7. In Figures $7(\mathrm{a})-7(\mathrm{c})$, it can be seen that the motion stability of the NSA becomes unstable with the $\% A$ increasing. Moreover, the motion stability of the NSA becomes unstable with the $\mu$ decreasing.

3.2. Simulation of Shock Response. There is no explicit analytical solution of equation (9). However, it can be solved numerically. From equation (9), it is found that the solution of the equation may be influenced by the natural frequency ratio $\Omega$, the shock excitation amplitude $\widetilde{F}$, damping ratio $\zeta$, 


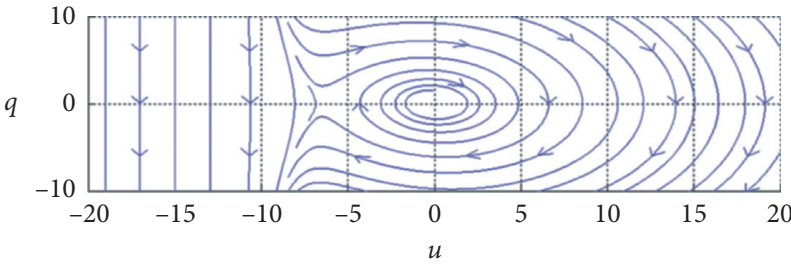

(a)

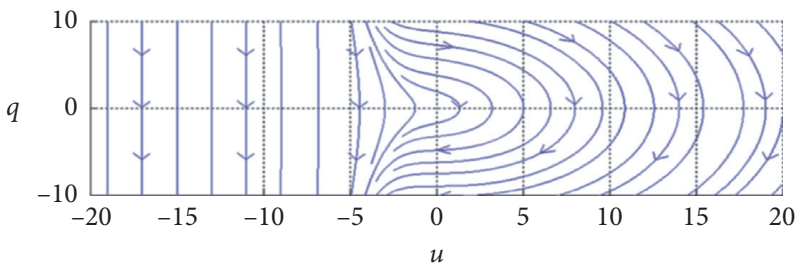

(c)

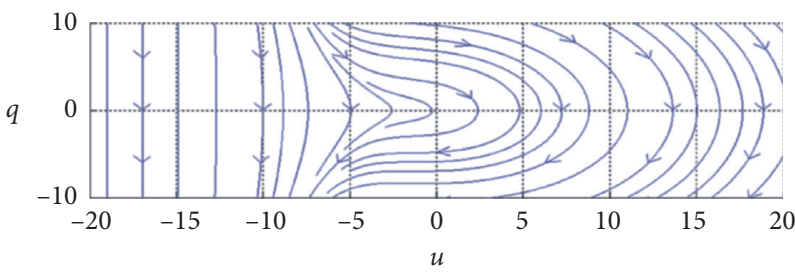

(e)

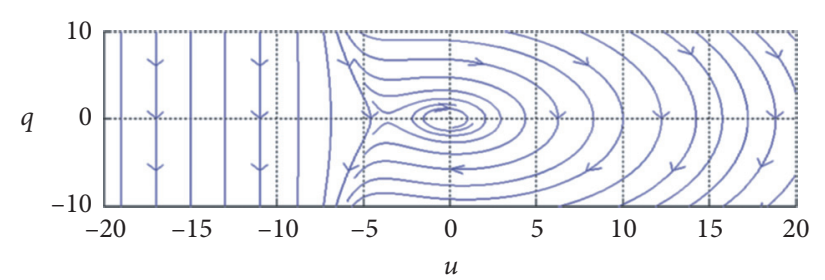

(b)

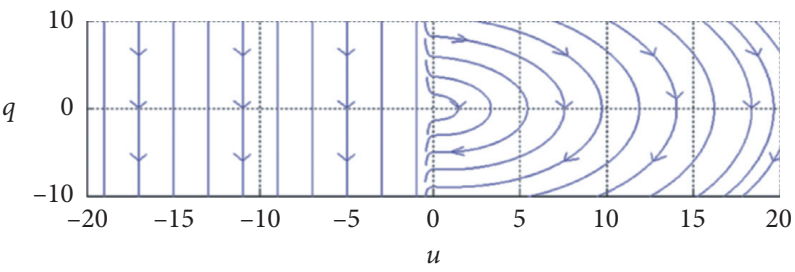

(d)

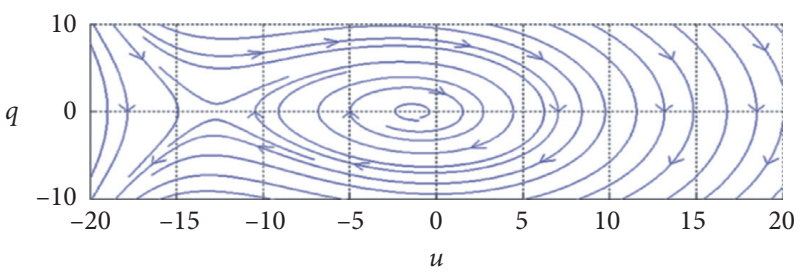

(f)

FIgURE 6: The shock excitation signal in the time domain.

and preload amplitude $\widetilde{A}$. The influence of these parameters on the shock response of the NSA is investigated in the following part.

The shock response spectrum of the NSA under different shock excitation amplitude $\widetilde{F}$ can be calculated numerically as shown in Figure 8(a). It reveals that the maximum acceleration response $u_{\max }$ " of the system increases with the shock amplitude $\widetilde{F}$ increasing when $\Omega<60 . u_{\max }{ }^{\prime \prime}$ increases under a specific $\widetilde{F}$ with the $\Omega$ decreasing when $\Omega<60$ as shown in Figure 8(a). In Figure 8(a), it is found that $u_{\max }{ }^{\prime \prime}$ is larger than $\widetilde{F}$ when $\Omega<6$, an amplification area; it is smaller than $\widetilde{F}$ when $\Omega>6$, an isolation area; and it is a constant value and equal to preload amplitude $\widetilde{A}$ when $\Omega>60$, an isolation static area. It also shows that the maximum acceleration response is equal to 0.5 when $\widetilde{F}=0.5$ because the shock excitation amplitude is below the preload amplitude; in this case, the preload force of the NSA can maintain the sensitive systems fixed to the foundation. In Figure 8(b), it can be found that the isolation static region of the NSA increases with $\widetilde{A}$ increasing. As the fitting value of $\mu$ increases, the frequency range of the isolation static region can be shortened as shown in Figure 8(c). It can be seen that the higher the damping ratio is, the lower the maximum acceleration response would be until $\Omega>60$ as shown in Figure 8(d).

The NSA is proposed to protect the sensitive systems from shock excitation so that it is expected to work in the isolation region. In Figure 8, the shock isolation performance of the NSA can be obtained when $\Omega>6$. The timedomain response of the NSA under different shock excitation is investigated when $\Omega=30, \Omega=50, \Omega=80$, and $\Omega=120$. The dimensionless resilient force-displacement curves of the NSA are shown in Figure 9. It shows that the linear stiffness of the NSA is hard when $\Omega$ is small and soft when $\Omega$ is higher. The NSA has a small range of the negative stiffness when the $\Omega$ is small.

The shock response of the NSA under different shock excitation amplitude $\widetilde{F}$ is shown in Figure 10. In Figure 10(a), it can be seen that the maximum acceleration response is constant and equal to the preload under different shock excitation amplitude $\widetilde{F}$ in terms of $\Omega=30, \Omega=50$, $\Omega=80$, and $\Omega=120$. For example, the time-domain response of the NSA when $\Omega=120$ is shown in Figure 11(a). However, in the case of $\Omega=30$, the maximum acceleration response is over the preload when $\widetilde{F}>13$, and the corresponding time-domain response is shown in Figure 11(c). In Figure 10(b), it is revealed that the maximum displacement response increases monotonically with the shock excitation amplitude increasing when $\Omega=30$ and $\Omega=50$ but there are two turning points in the curve when $\Omega=80$ and $\Omega=120$. The corresponding time-domain displacement response is shown in Figures 11(b) and 11(d), respectively. It shows that the maximum acceleration response increases and the maximum displacement response decreases with the damping ratio increasing.

In Figures 11(b) and 11(f), it is shown that the maximum displacement responses and the actual response frequency of the NSA are similar when the NSA is subjected to $\widetilde{F}=3.65$ and $\widetilde{F}=13$. It is a jump phenomenon of a system with nonlinear stiffness. To illustrate the jump phenomenon, a 


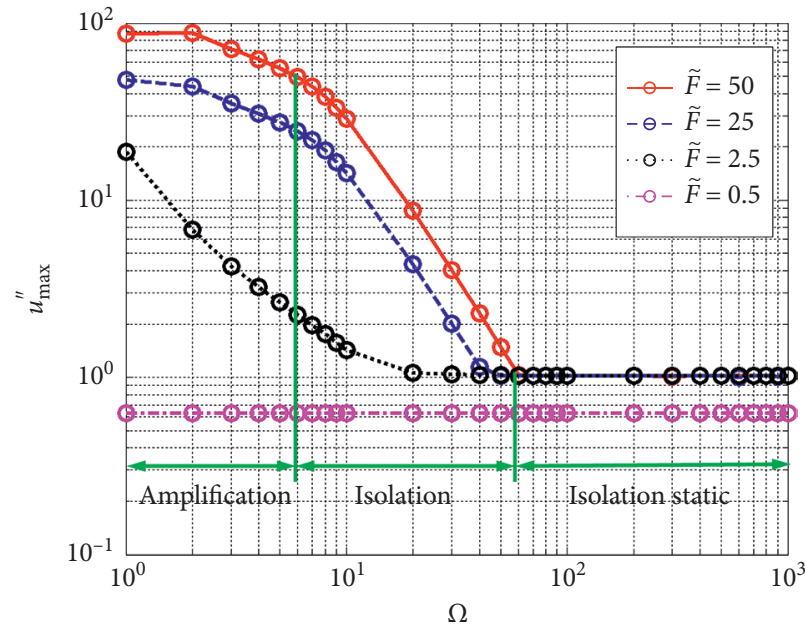

(a)

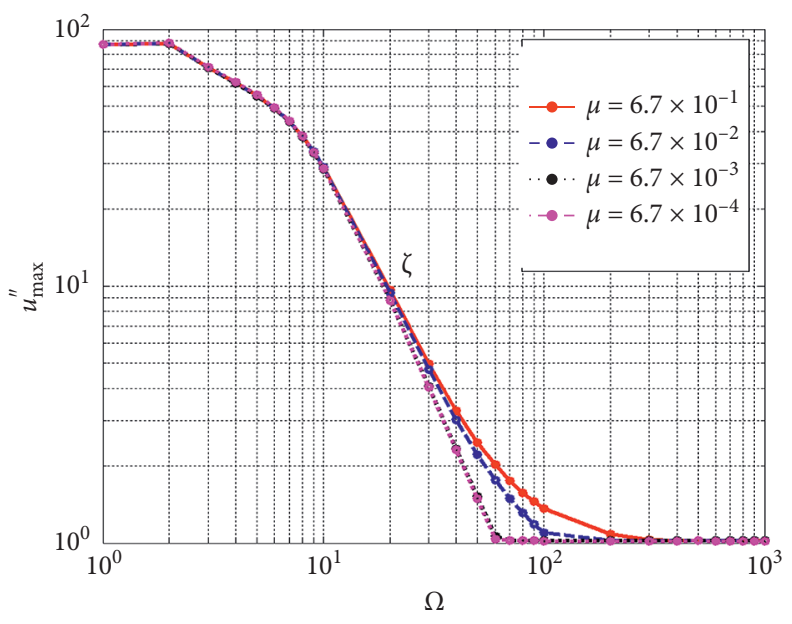

(c)

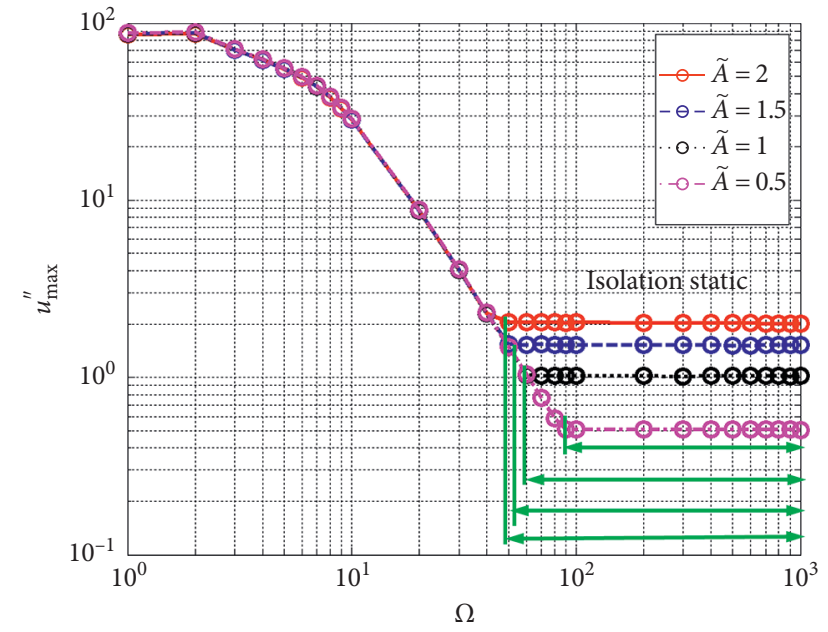

(b)

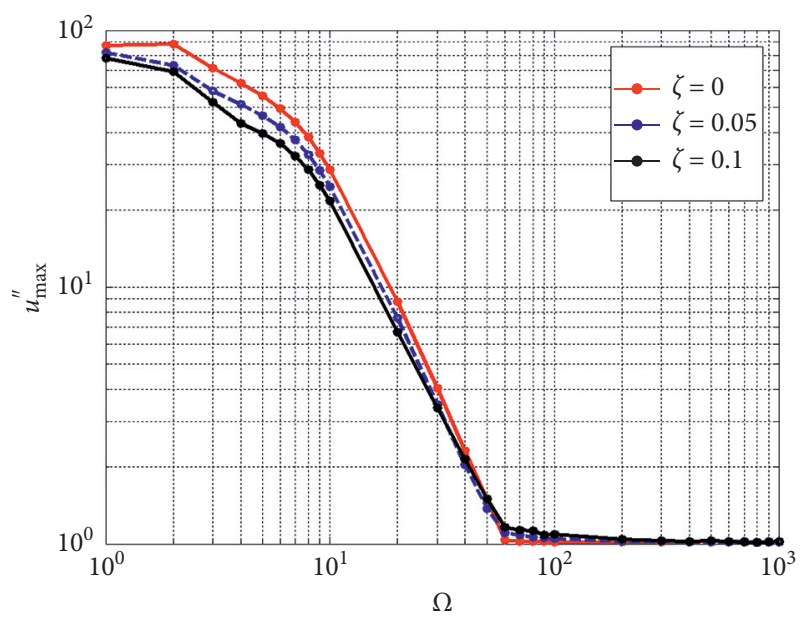

(d)

FigURE 7: The streamslice of the NSA with different values of the $\% A$ and $\mu(\zeta=0.05$ ). (a) $\widetilde{A}=0.01, \mu=1$. (b) $\widetilde{A}=0.1, \mu=1$. (c) $\widetilde{A}=1, \mu=1$. (d) $\widetilde{A}=1, \mu=0.1$. (e) $\widetilde{A}=1, \mu=2$. (f) $\widetilde{A}=1, \mu=5$.

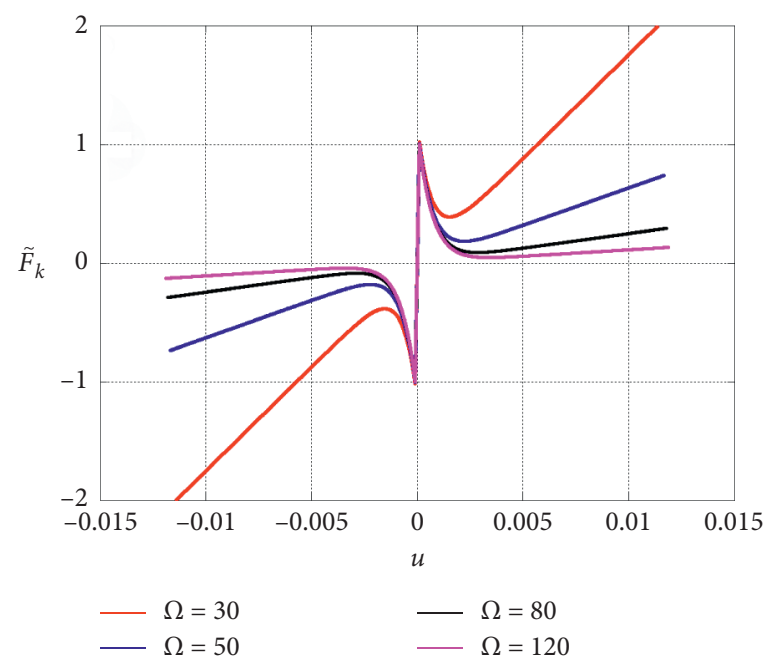

Figure 8: Absolute acceleration shock spectrum of the NSA. (a) Different shock excitation amplitude $\% F, \% A=1, \mu=6.7 \times 10^{-4}, \zeta=0$. (b) Different preload amplitude $\% A=1, \mu=6.7 \times 10^{-4}, \% F=50, \zeta=0$. (c) Different fitting values $\mu, \% A=1, \% F=50, \zeta=0$. (d) Different damping ratio $\mu=6.7 \times 10^{-4}, \% A=1, \% F=50(w=200 \pi / \mathrm{s})$. 

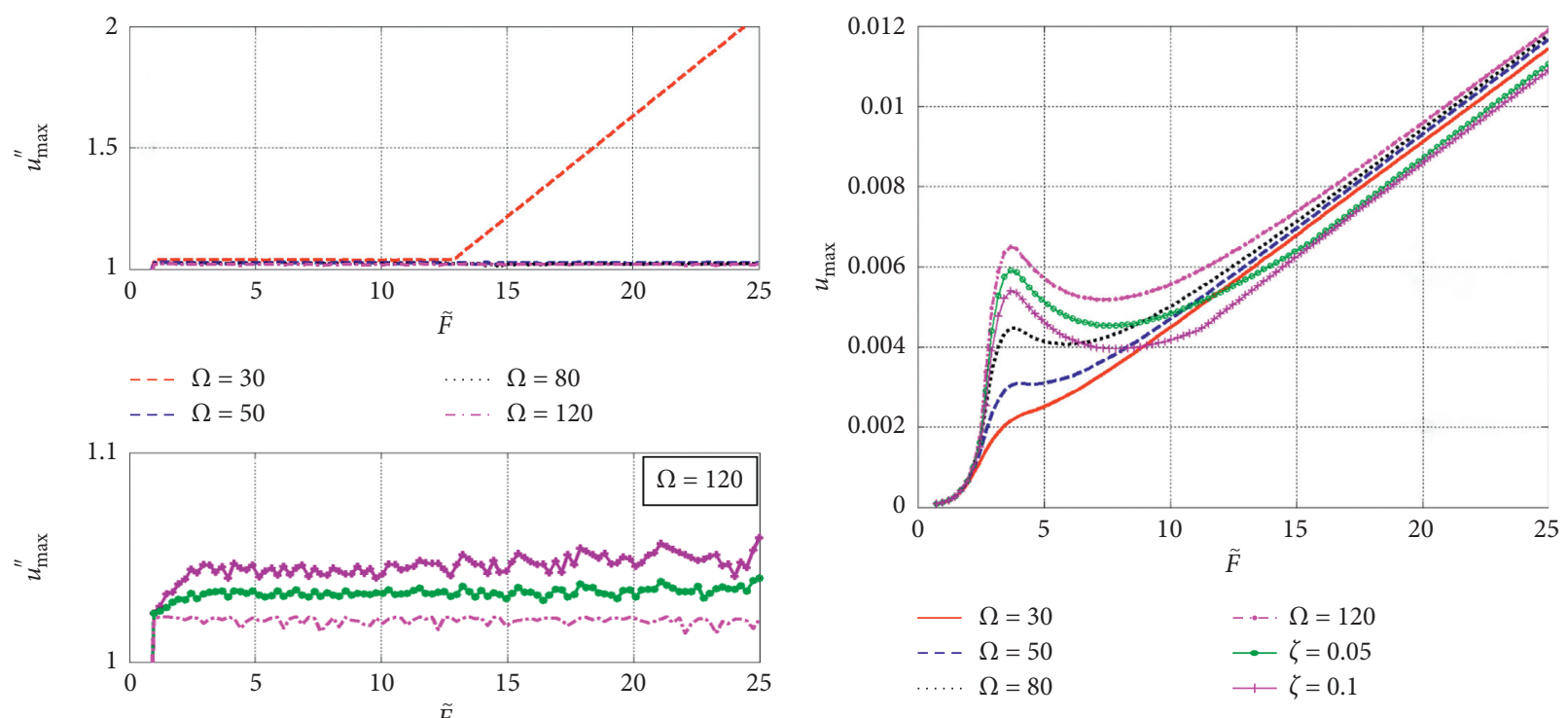

(a)

(b)

FIgURE 9: The dimensionless resilient force-displacement curves of the NSA $\left(\zeta=0, \widetilde{A}=1, w=200 \pi / \mathrm{s}, \mu=6.7 \times 10^{-4}\right)$.

frequency ratio $\Omega_{1}$ is defined to calculate the frequency response function. The $\Omega_{1}$ is expressed

$$
\Omega_{1}=\frac{w}{w_{1}},
$$

where $w_{1}$ represents the actual response frequency of the NSA under shock excitation. The relation of shock excitation amplitude $\widetilde{F}$ and $\Omega_{1}$ can be calculated as shown in Figure 12. It can be seen that the jump phenomenon happens when linear stiffness of the NSA is soft, such as $\Omega=120$. The unstable points as shown in Figure 12(a) can be judged by equations (17) and (18). It can be found that $\Omega_{1}$ is closed to the value of $\Omega$ when $\widetilde{F}$ is higher enough as shown in Figure 12(a).

The influence of the preload amplitude and damping ratio on the unstable region is investigated as shown in Figures 12 (b) and 12 (c), respectively. It shows that the higher value of preload amplitude, such as $\widetilde{A}=2$, can lead to a wider range of motion instability, shown in Figure 12(b), because the negative stiffness region is wider in this case. Besides, the increase of damping ratio can put the jump point forward as shown in Figure 12(c).

3.3. Comparison of Different Preload Structures. To evaluate the advantage of the NSA, the shock response of the NSA is calculated compared to preload structure with positive stiffness (PSPS) and linear stiffness system (LSS). The NSA, PSPS, and LSS considered here have the same linear positive stiffness and preload force. The calculation results are shown in Figure 13.
In Figure 13, the shock acceleration response of different systems is shown. And it is found that the NSA has a better isolation performance compared to PSPS when the linear positive stiffness is soft as shown in Figures 13(a) and 13(a). The maximum acceleration response of the NSA is equal to the preload force and the average value of acceleration is much lower than PPS. Although the NSA and PSPS have the same preload force and linear stiffness, the response frequency of the PNS is lower than PSPS and closed to LSS due to the global negative stiffness. The maximum displacement response of the NSA is slightly higher than PSPS as shown in Figures 13(d) and 13(e). The maximum acceleration response can exceed the value of preload force when the linear stiffness is hard as shown in Figure 13(c), but it is still lower than the PSPS.

In Figure 13, it is shown that the shock response of the NSA is closed to LSS globally, but it is quite different around the equilibrium position $(x=0)$ because of the preload force and the global negative stiffness. Although the maximum displacement of the NSA is slightly higher than PPS, the shock isolation performance of the NSA is better than PSPS due to the fact that the maximum acceleration response is always lower. Moreover, the maximum acceleration response is below the preload force when the linear stiffness is soft.

To evaluate the damage level of the sensitive systems installed on the shock absorber, the shock response spectrum (SRS) was used to analyze the shock isolation performance [2]. The SRS is calculated by the acceleration response curve shown in Figure 13. The SRS of the NSA is compared to the PSPS and LSS as shown in Figure 14. 


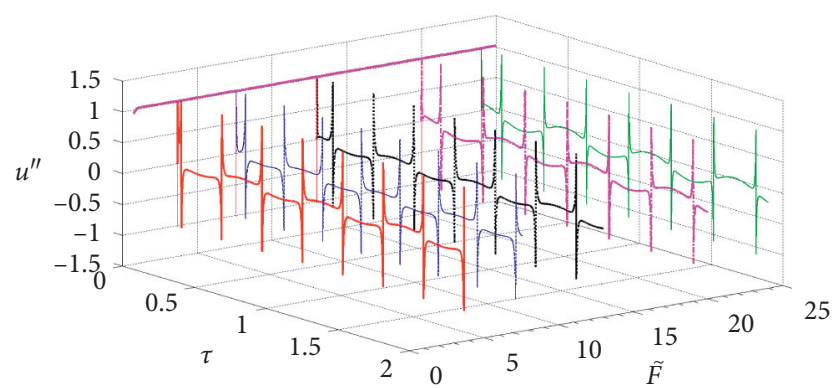

(a)

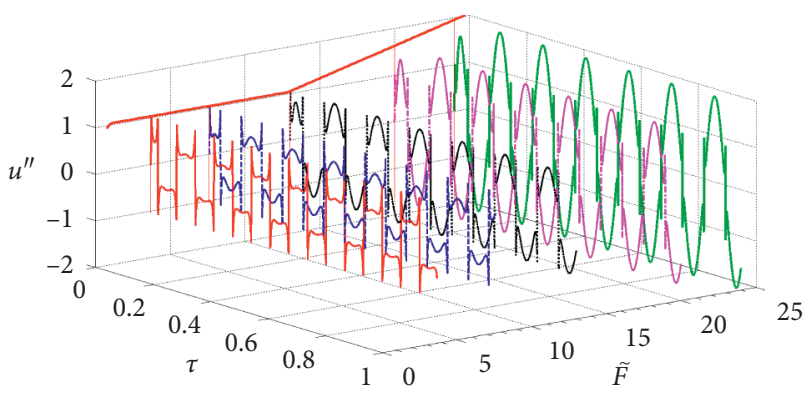

(c)

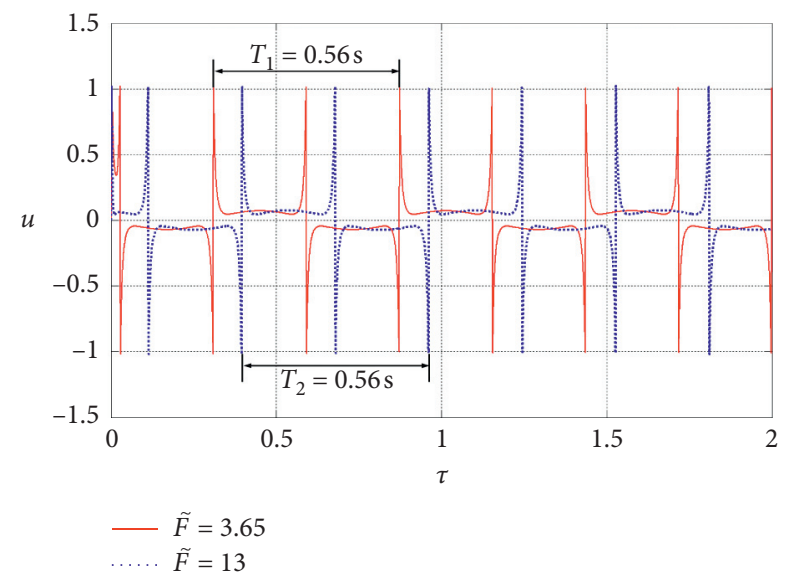

(e)

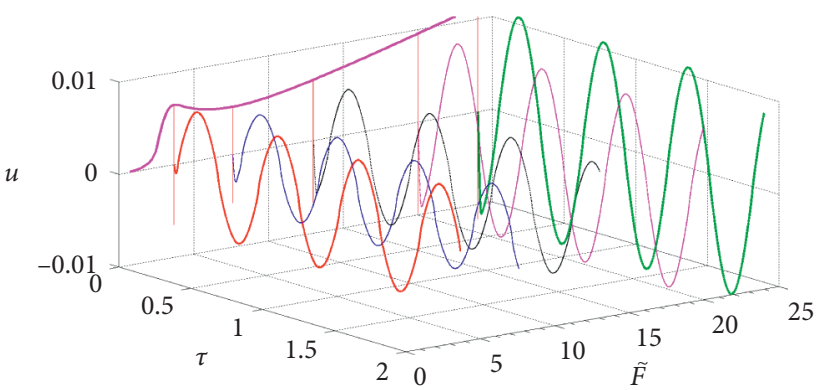

(b)

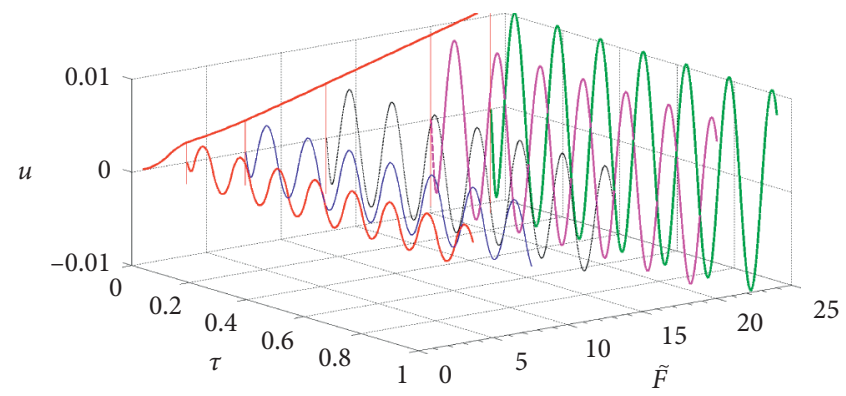

(d)

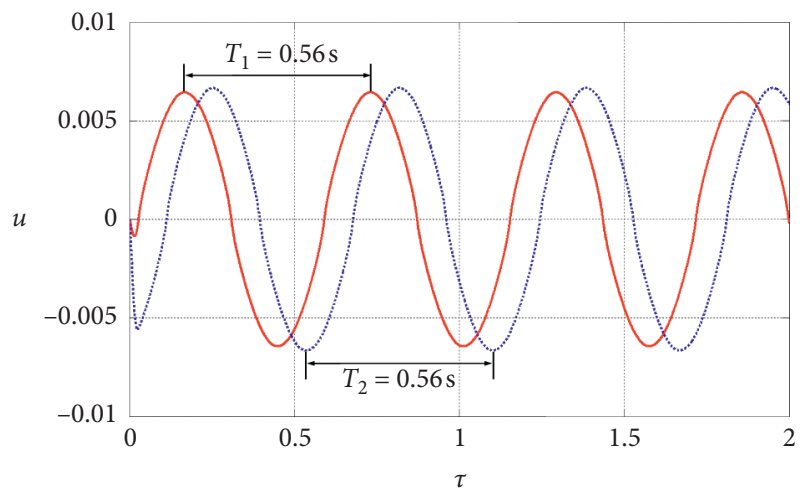

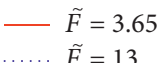

(f)

Figure 10: The influences of different $\Omega$ on shock response of the NSA $\left(\widetilde{A}=1, w=200 \pi / s, \mu=6.7 \times 10^{-4}\right)$. (a) Absolute acceleration response. (b) The relative displacement response.

In Figure 14(a), it is shown that the SRS of the NSA is smaller than the PSPS when the natural frequency is over $3 \mathrm{~Hz}$. The SRS of the NSA is closed to LSS in the lowfrequency region but it is higher than LSS in the highfrequency region. Besides, the SRS of the NSA is less than the PSPS when the natural frequency is over $7 \mathrm{~Hz}$ as shown in Figure 14(b). However, the SRS of the NSA slightly less than the PSPS when the natural frequency is more than $10.2 \mathrm{~Hz}$ as shown in Figure 14(c). From the above, it can be concluded that PNS has a better shock isolation performance compared to PSPS and LS, even though the linear stiffness is hard.

\section{Shock Test}

The prototype of the NSA is fabricated and tested by a drop table. The permanent magnets and the cylindrical coil spring are the key components of the NSA. Before the shock test, the static characteristic of the key components is tested by static testing platform WANCE-ETM503 $\mathrm{A}$ as shown in Figures $3(\mathrm{a})$ to $3(\mathrm{c})$.

The material of the permanent magnets is Neodymium, which is the most important negative stiffness component of the NSA. Since the permanent magnets are brittle material, it can be broken up under the rigid collision. Therefore, a 


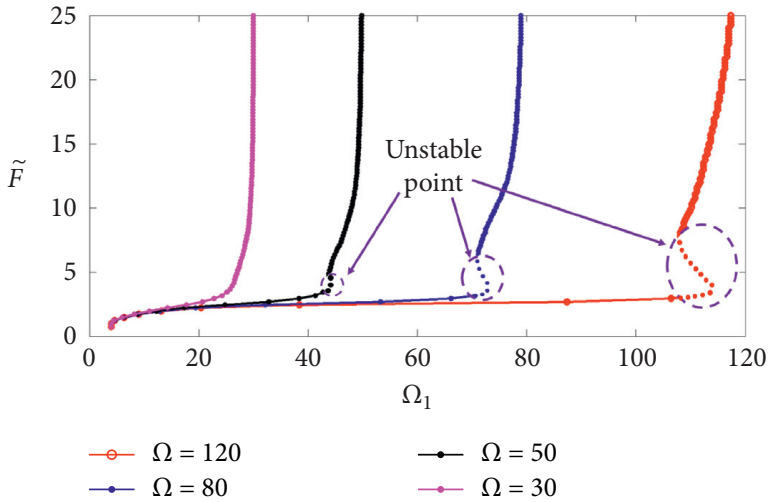

(a)

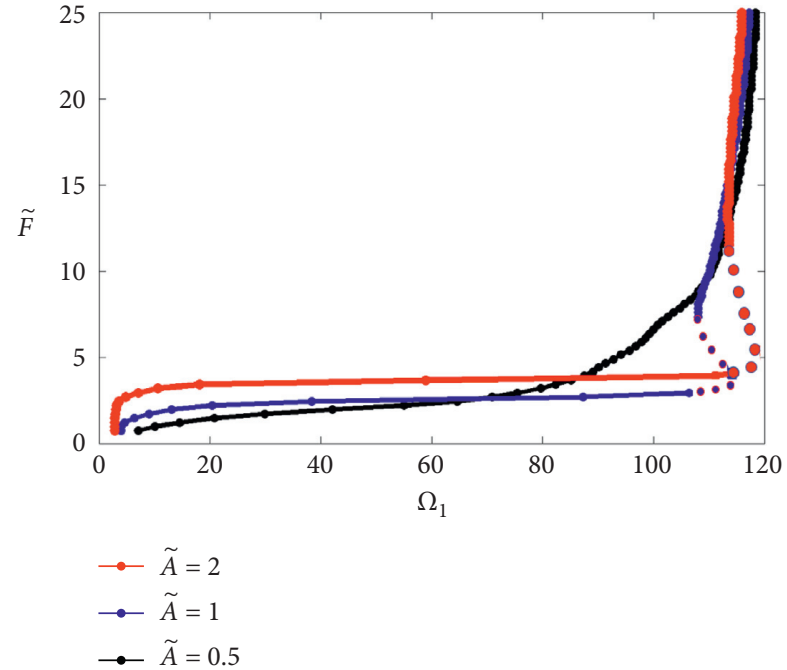

(b)

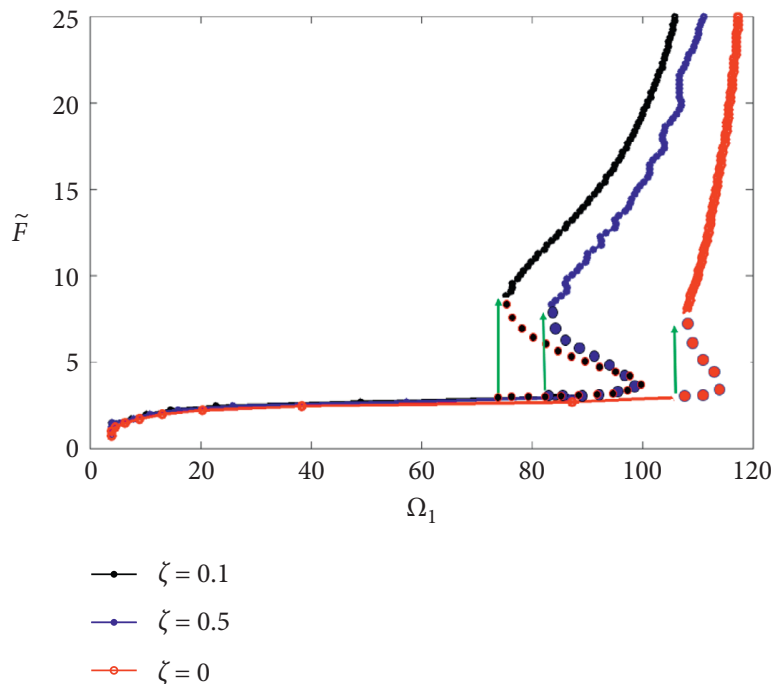

(c)

Figure 11: The time-domain response of the NSA $\left(\widetilde{A}=1, w=200 \pi / \mathrm{s}, \mu=6.7 \times 10^{-4} \zeta=0\right)$. (a) Shock acceleration response under different $\% F, \Omega=120$. (b) Shock displacement response under different $\% F, \Omega=120$. (c) Shock acceleration response under different $\% F, \Omega=30$, $\zeta=0$. (d) Shock displacement response under different $\% F, \Omega=30$. (e) Shock acceleration response when $\% F=3.65$ and $\% F=13, \Omega=120$. (f) Shock displacement response when $\% F=3.65$ and $\% F=13, \Omega=120$.

rubber washer is placed on the contact face of the lower magnet, as shown in Figure 3(b). The thickness of the rubber washer is $2 \mathrm{~mm}$. It shows that the maximum attractive force $F_{\mathrm{m}}$ between permanent magnets set is $37 \mathrm{~N}$ when the $n=1$, and it is $111 \mathrm{~N}$ when the $n=3$ as shown in Figure 3(b). Two linear springs with different stiffness are considered here, the resilient force $F_{L}$ of the spring is as shown in Figure 3(e), used to interact with permanent magnets sets $(n=3)$, and the total resilient force $F_{\mathrm{k}}$, the product of $F_{\mathrm{m}}$ and $F_{L}$, is shown in Figure 3(f).

A typical drop table machine (SY10-500) is used to simulate the shock excitation as shown in Figure 6. The prototype of the NSA with different linear stiffness is mounted on the drop table as shown in Figure 15. The acceleration sensor B\&K-4384 used to measure the acceleration signal is the product of Brüel and Kjær company.

Three different levels of shock excitation generated by different drop heights are used to test the NSA as shown in Figures 16(a)-16(c). It is shown that the corresponding measured maximum acceleration peaks are $15 \mathrm{~g}, 28 \mathrm{~g}$, and $46 \mathrm{~g}$, respectively. The testing results of shock response are shown in Figures 17(a)-17(f). Figures 17(a)-17(c) show the shock acceleration response of the NSA with the soft linear stiffness under different shock excitation. The weight of the mass used to simulate the sensitive system is $3.7 \mathrm{~kg}$ so that the mass can move while the external shock acceleration is over $3 \mathrm{~g}$. It can be found that the maximum acceleration 


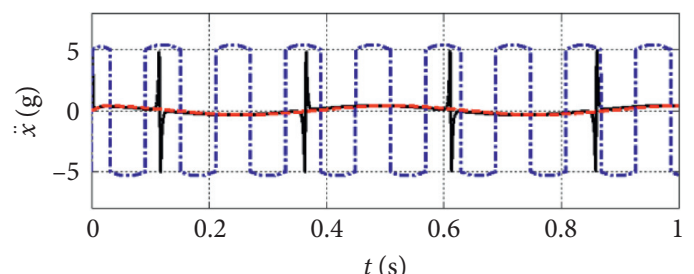

$$
\begin{aligned}
& \text { _ NSA } \\
& \text { - - PSPS } \\
& \text { - - LSS }
\end{aligned}
$$

(a)

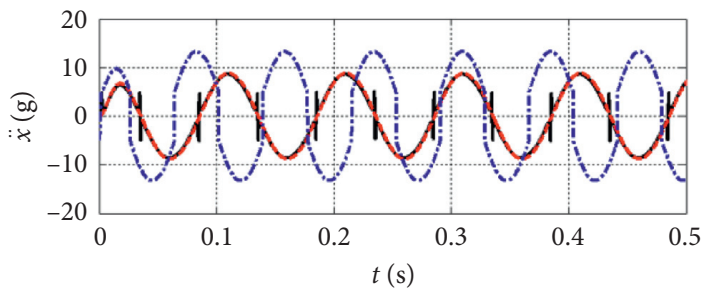

- NSA

-.. PSPS

- - LSS

(c)

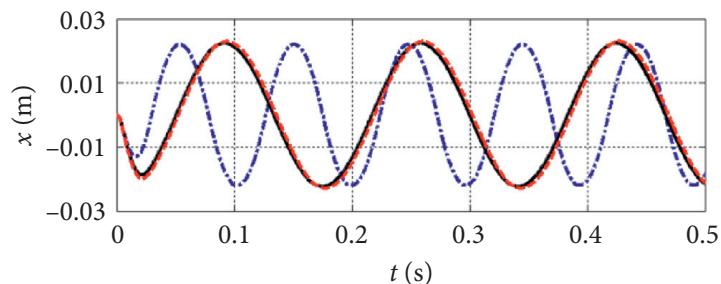

_ NSA
-. PSPS
- LSS

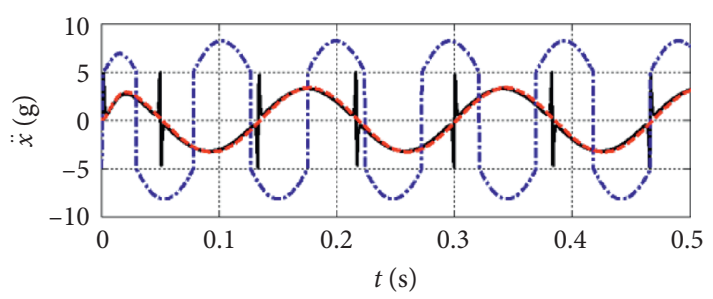

- NSA

-. - PSPS

- - - LSS

(b)

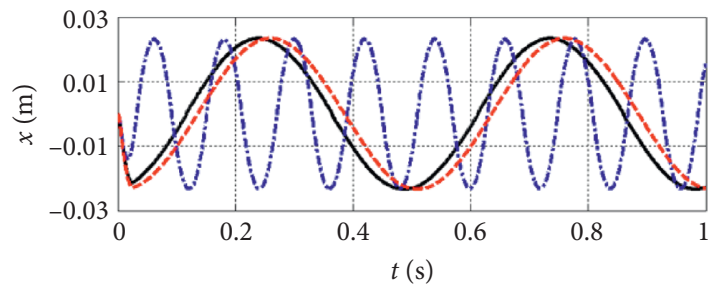

- NSA

-.- LSS

(d)

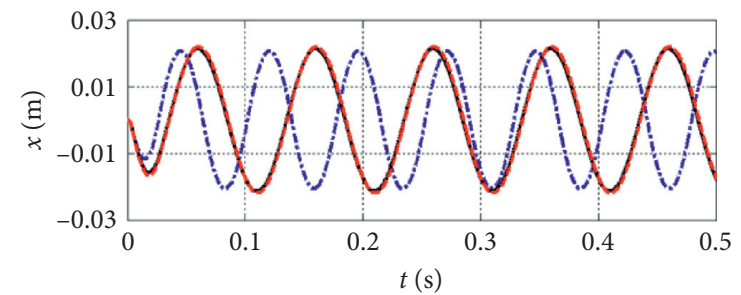

- NSA

...- PSPS

_. LSS

(e)

(f)

Figure 12: The frequency response of the NSA $\left(w=200 \pi / \mathrm{s}, \mu=6.7 \times 10^{-4}\right)$. (a) Different frequency ratio $\Omega, \zeta=0, \widetilde{A}=1$. (b) Different damping ratio $\zeta, \Omega=120, \widetilde{A}=1$. (c) Different preload amplitude $\widetilde{A}$.

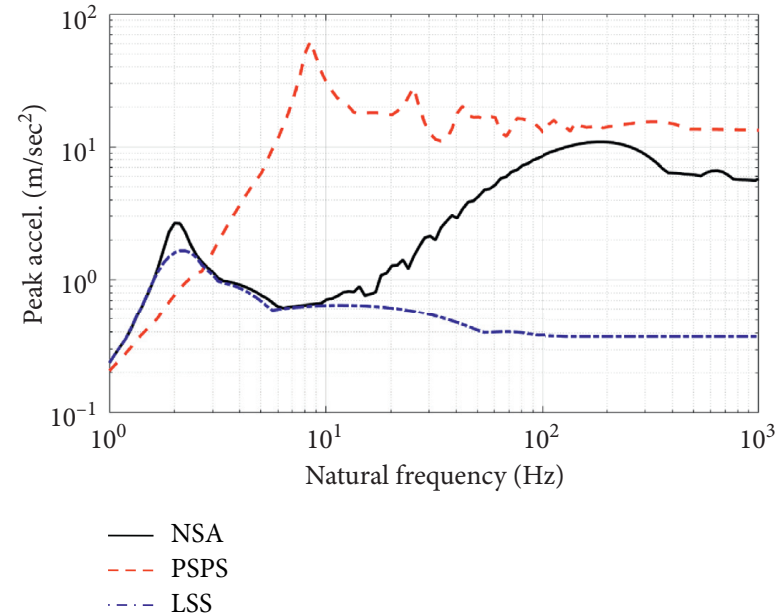

(a)

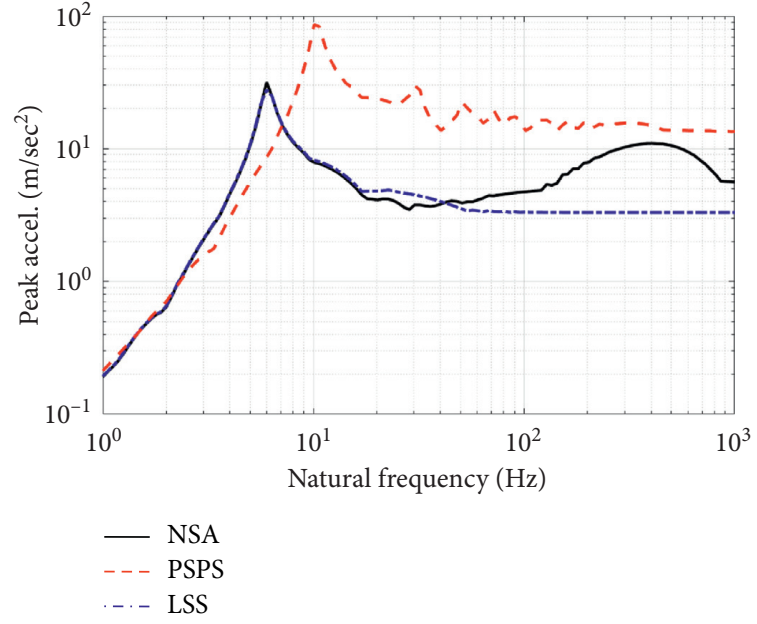

(b)

FIgURE 13: Continued. 


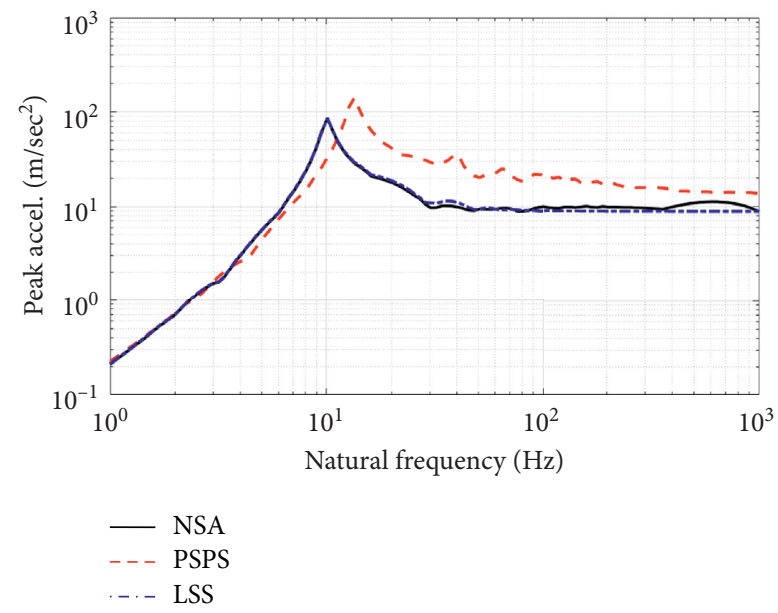

(c)

Figure 13: The shock response of the systems $\left(\mu=6.7 \times 10^{-4}, m=1 \mathrm{~kg}, n=1, A=5 N, F=50 N, w=200 \pi / \mathrm{s}\right)$. (a) $\Omega=100$. (b) $\Omega=30$. (c) $\Omega=20$. (d) (e) (f)

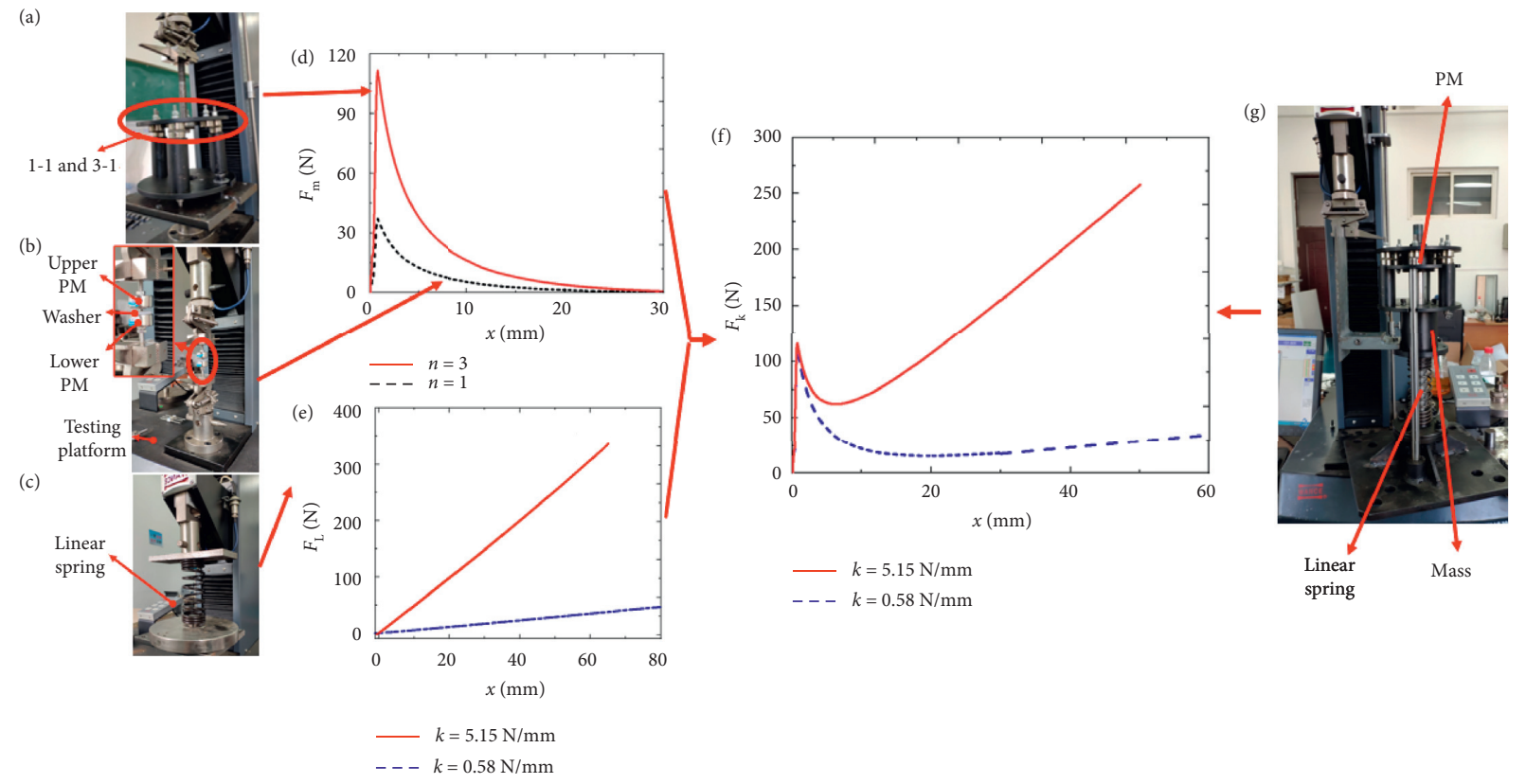

FIGURE 14: The SRS of the different systems. (a) $\Omega=100$. (b) $\Omega=30$. (c) $\Omega=20$.

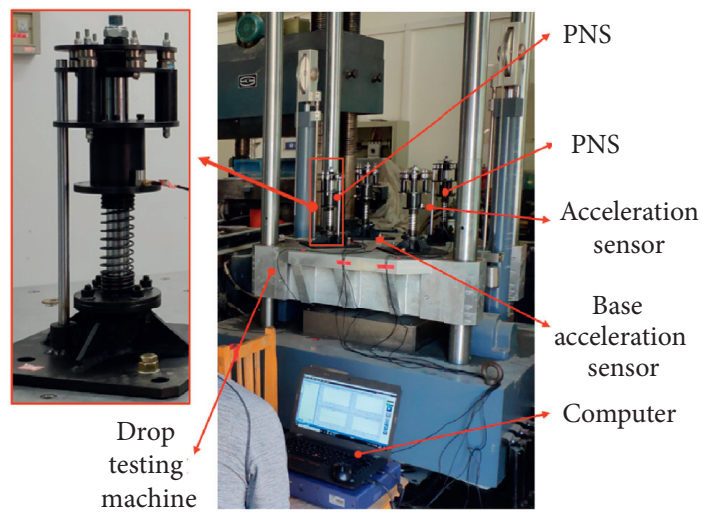

FIGURE 15: The shock testing platform. 


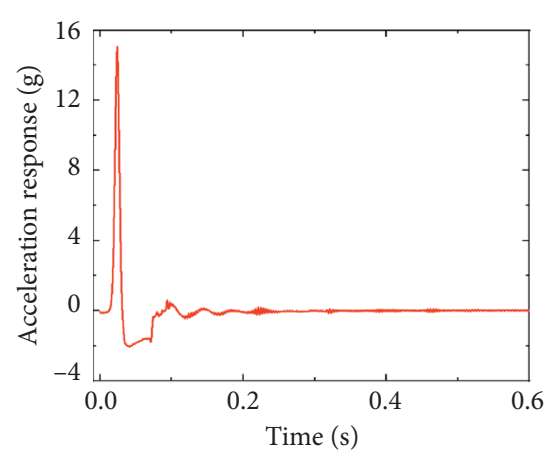

$-90 \mathrm{~mm}$

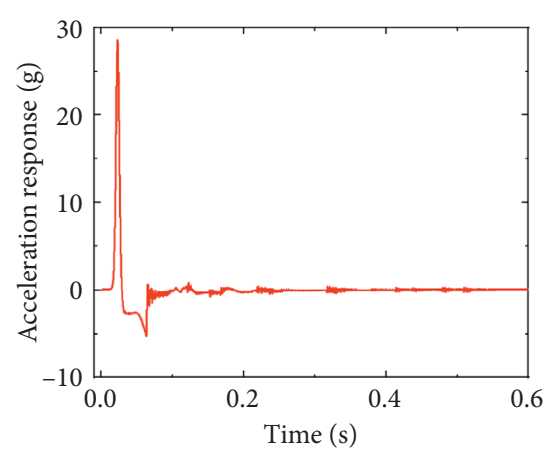

$-120 \mathrm{~mm}$

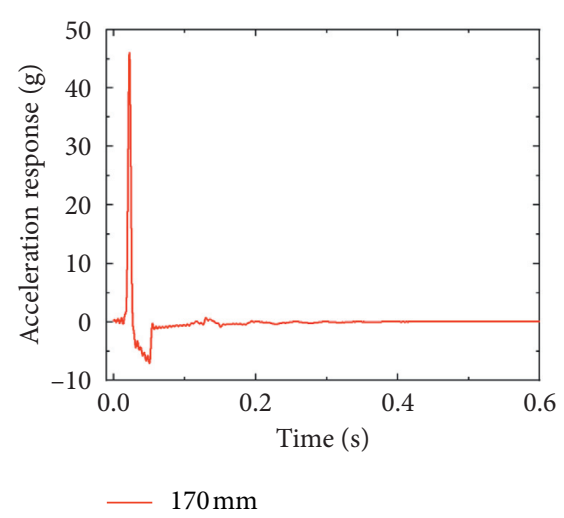

(c)

Figure 16: The signal input from different drop heights. (a) The drop height is $90 \mathrm{~mm}$. (b) The drop height is $120 \mathrm{~mm}$. (c) The drop height is $170 \mathrm{~mm}$.

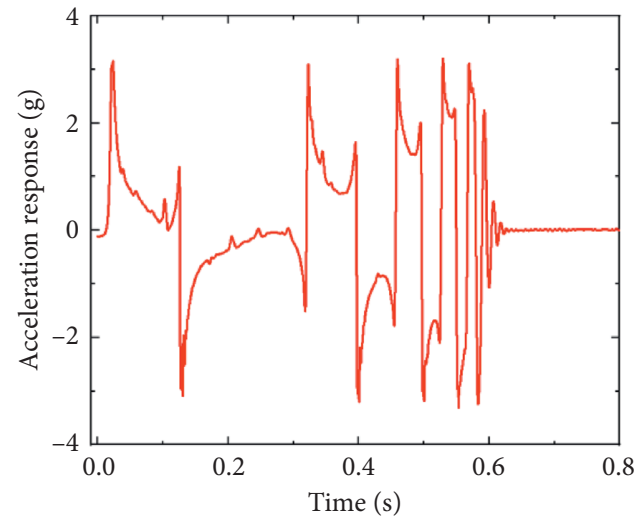

$-90 \mathrm{~mm}$

(a)

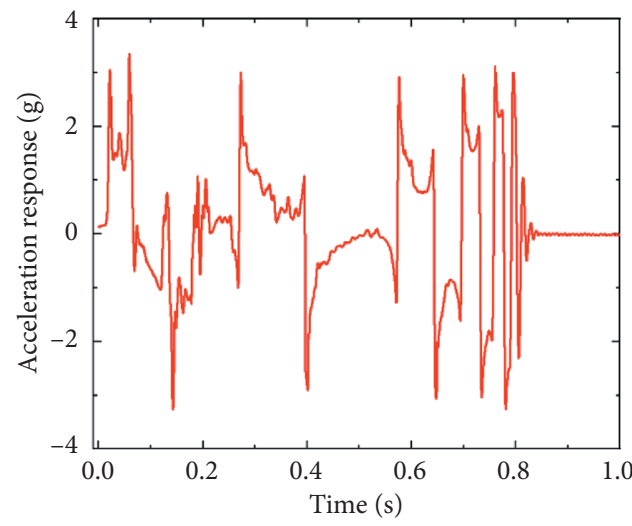

$-170 \mathrm{~mm}$

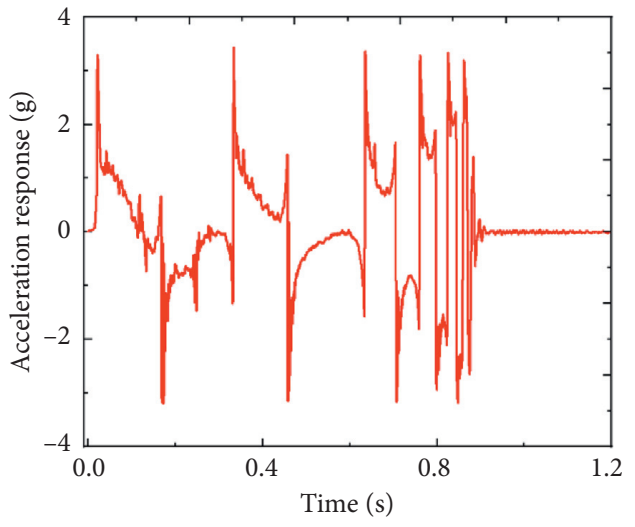

$-120 \mathrm{~mm}$

(b)

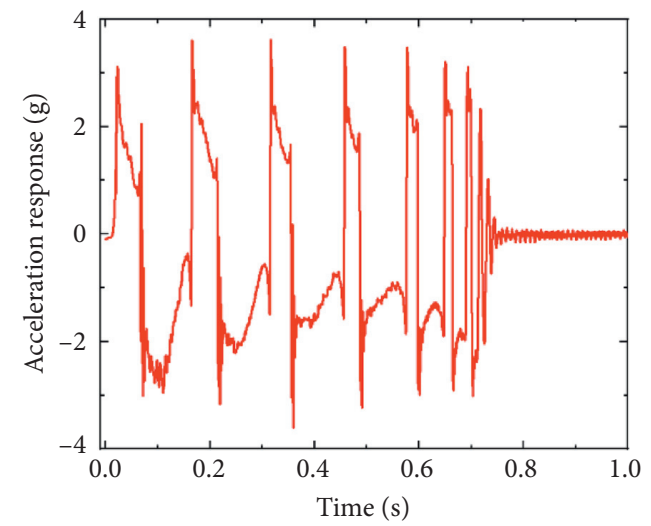

$-90 \mathrm{~mm}$

(c)

FIGURE 17: Continued.

(d) 


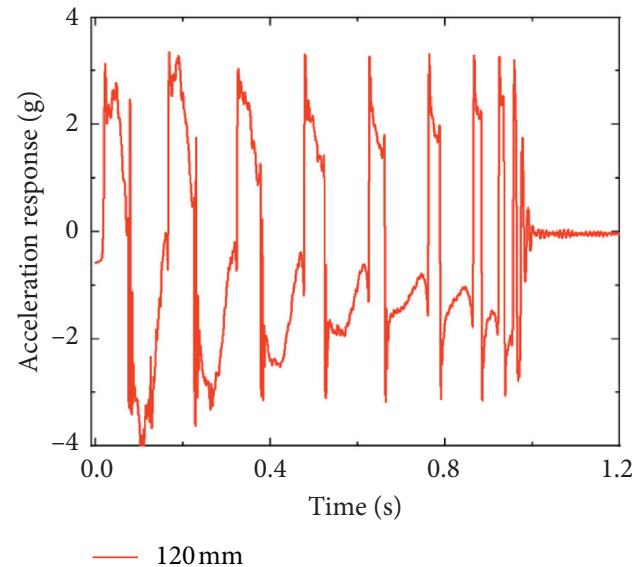

(e)

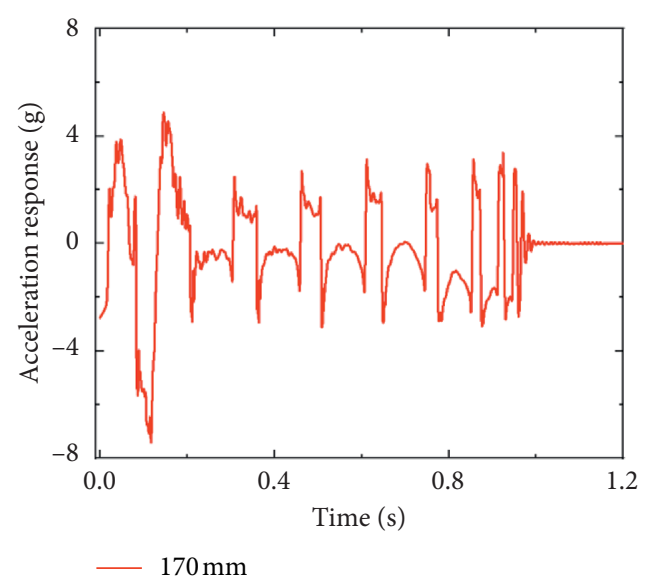

(f)

FIgURE 17: The shock testing results of the NSA under different drop heights. (a) The drop height is $90 \mathrm{~mm}$ when $k=0.58 \mathrm{~N} / \mathrm{mm}$. (b) The drop height is $120 \mathrm{~mm}$ when $k=0.58 \mathrm{~N} / \mathrm{mm}$. (c) The drop height is $170 \mathrm{~mm}$ when $k=0.58 \mathrm{~N} / \mathrm{mm}$. (d) The drop height is $90 \mathrm{~mm}$ when $k=5.15 \mathrm{~N} / \mathrm{mm}$. (e) The drop height is $120 \mathrm{~mm}$ when $k=5.15 \mathrm{~N} / \mathrm{mm}$. (f) The drop height is $170 \mathrm{~mm}$ when $k=5.15 \mathrm{~N} / \mathrm{mm}$.

response is always equal to preload. However, the maximum acceleration response of the NSA with hard linear stiffness is over the preload and increases with the drop height increasing.

From the above, it is concluded that the NSA has the advantages of shock isolation and the acceleration response is always less than the safety limit under different shock levels when the NSA with a soft linear stiffness. These conclusions are in agreement with the simulation results in Section 3.

\section{Conclusion}

In this paper, the NSA has been presented and discussed. The aim of the NSA is proposed to protect the sensitive systems in a complex shock environment. The NSA has a preload structure used to maintain the sensitive systems in a fixed relationship with the supporting structure and it can be released to allow relative movement for shock isolation the same as the PSPS. Moreover, The NSA has a global negative stiffness characteristic that differs from the PSPS and LS.

Global negative stiffness of the NSA is influenced by the attractive force generated by permanent magnet sets. Region of negative stiffness is increased with the attractive force increasing and a jump phenomenon would become obvious when the region of negative stiffness is large. Although the global negative stiffness can cause a jump phenomenon around the equilibrium position, the NSA has better shock isolation performance than others because of the global negative stiffness. In addition, a proper soft linear stiffness can improve the shock performance of the NSA, because it can ensure that the maximum resilience of the NSA is always below the preload force under different shock excitation levels.

From the shock test results, it is verified that the NSA has good shock performance. And the simple structure of the
NSA is advantageous to the application for sensitive equipment exposed to a shock environment.

\section{Data Availability}

The data used to support the findings of this study are included within the article.

\section{Conflicts of Interest}

The authors declare that they have no conflicts of interest.

\section{Acknowledgments}

This project was supported by the National Basic Research Program of China (613157010102).

\section{References}

[1] S. Goepfert, T. Braman, and D. Hagenson, "Elastomeric vibration and shock isolation for inertial sensor assemblies," pp. 8-5, 2004, U. S. Patent Application 10/354, 536.

[2] B. W. Li and Q. M. Li, "Damage boundary of structural components under shock environment," International Journal of Impact Engineering, vol. 118, pp. 67-77, 2018.

[3] T. D. Barber, K. Young, and T. Hindle, "Launch lock assemblies with reduced preload and spacecraft isolation systems including the same," pp. 10-25, 2016, U. S. Patent 9, 475, 594.

[4] H. M. Sedighi, K. H. Shirazi, A. Reza, and J. Zare, "Accurate modeling of preload discontinuity in the analytical approach of the nonlinear free vibration of beams," in Proceedings of the Institution of Mechanical Engineers, Part C: Journal of Mechanical Engineering Science, vol. 226, no. 10, pp. 2474-2484, 2012.

[5] S. Heimbs, S. Heller, P. Middendorf et al., "Low velocity shock on CFRP plates with compressive preload: test and modelling," International Journal of Shock Engineering, vol. 36, no. 10-11, pp. 1182-1193, 2009. 
[6] M. A. Garcia-Romeu-Martinez, M. A. Sek, and V. A. CloquellBallester, "Effect of initial pre-compression of corrugated paperboard cushions on shock attenuation characteristics in repetitive shocks," Packaging Technology and Science: An International Journal, vol. 22, no. 6, pp. 323-334, 2009.

[7] R. A. Ibrahim, "Recent advances in nonlinear passive vibration isolators," Journal of Sound and Vibration, vol. 314, no. 35, pp. 371-452, 2008.

[8] J. Zhou, Q. Xiao, D. Xu, H. Ouyang, and Y. Li, "A novel quasizero-stiffness strut and its applications in six-degree-offreedom vibration isolation platform," Journal of Sound and Vibration, vol. 394, pp. 59-74, 2017.

[9] X. Liu, X. Huang, and H. Hua, "On the characteristics of a quasi-zero stiffness isolator using Euler buckled beam as negative stiffness corrector," Journal of Sound and Vibration, vol. 332 , no. 14 , pp. 3359-3376, 2013.

[10] D. Vokoun, G. Tomassetti, M. Beleggia, and I. Stachiv, "Magnetic forces between arrays of cylindrical permanent magnets," Journal of Magnetism and Magnetic Materials, vol. 323 , no. 1, pp. 55-60, 2011.

[11] N. C. Shekhar, H. Hatwal, and A. K. Mallik, "Response of nonlinear dissipative shock isolators," Journal of Sound and Vibration, vol. 214, no. 4, pp. 589-603, 1998.

[12] Y. Ma, M. He, W. Shen, and G. Ren, "A planar shock isolation system with high-static-low-dynamic-stiffness characteristic based on cables," Journal of Sound and Vibration, vol. 358, pp. 267-284, 2015.

[13] C. M. Harris and A. G. Piersol, Harris' Shock and Vibration handbook, McGraw-Hill, New York, NY, USA, 2002. 